\title{
Experiences with regular e-transfer of geodetic INTENSIVE data at Wettzell
}

\section{A. Neidhardt}

Forschungseinrichtung Satellitengeodäsie, Technische Universität München,

Geodätisches Observatorium Wettzell

Sackenrieder Str. 25, D-93444 Bad Kötzting, Germany

\section{G. Kronschnabl*, C. Plötz, R. Dassing}

Bundesamt für Kartographie und Geodäsie, Geodätisches Observatorium Wettzell Sackenrieder Str. 25, D-93444 Bad Kötzting, Germany

\section{W. Schwegmann}

Bundesamt für Kartographie und Geodäsie

Richard-Strauss-Allee 11, D-60598 Frankfurt am Main, Germany

\section{Thorand, G. Engelhardt}

Bundesamt für Kartographie und Geodäsie

Karl-Rothe-Straße 10-14, D-04105 Leipzig, Germany

For several years the radio telescope of the Geodetic Observatory Wettzell has participated in the daily one-hour INTENSIVE-sessions (INT), in addition to the 24-hour sessions, in order to determine UT1-UTC. The INT1 sessions are observed together with Kokee Park Geophysical Observatory (Hawaii, USA) and partly with Svetloe (Russia) every weekday and are correlated at Washington Correlator. On Saturday and Sunday, Wettzell is engaged together with Tsukuba (Japan) in the INT2 sessions which are correlated in Japan. On Monday morning INT3 session, which is correlated in Bonn, completes the INTENSIVE observations in the network of Wettzell, Tsukuba and Ny Ålesund. For all these INTENSIVE experiments, e-transfer is used to ship the data over standard internet connections to the correlators in near real-time. Wettzell itself routinely uses a fast internet connection at $622 \mathrm{Mbit} / \mathrm{sec}$ for these e-transfers. All these efforts reduce the delay times from taking the observations, to correlation, to publishing the final geodetic data product at the primary IVS Data Centers.

Science and Technology of Long Baseline Real-Time Interferometry:

The 8th International e-VLBI Workshop, EXPReSO9

June 22 - 262009

Madrid, Spain

\footnotetext{
* Speaker.
} 


\section{Introduction}

In times of global warming and climate change, geodetic data have become more and more important in defining and maintaining reference frames for earth observations and practical applications such as high-precision navigation and positioning. The International VLBI Service for Geodesy and Astrometry (IVS) therefore offers appropriate data products derived from VLBI observations with a global network of radio telescopes. Besides the contribution to the celestial reference frame and the monitoring of the coordinates of the celestial pole (nutation and precession), geodetic VLBI contributes uniquely to the monitoring of universal time (UT1) and the length of day (LOD) [1]. In order to determine UT1-UTC, regular daily one-hour sessions (INTENSIVES) are scheduled in addition to the regular 24-hour sessions. The radio telescope Wettzell (RTW) has a key role because it participates in all INTENSIVE experiments. With e-transfer, where the data are shipped immediately after the experiment to the correlator, the production times from the observation to the final geodetic data product are considerably reduced.

\section{The INTENSIVES round trip}

The round trip for observing geodetic data starts and ends at one of the three primary data centers of the IVS: Crustal Dynamics Data Information System (CDDIS) in the USA, Paris Observatory (OPAR) in France and Bundesamt für Kartographie und Geodäsie Leipzig (BKG) in Germany. These data centers mirror each other several times a day to ensure common data stocks. They offer the schedule files prepared by the IVS Operation Centers for download by the IVS Network Station, they receive the log and ancillary data files after the observations from the IVS Network Stations, and they archive data products from the IVS Analysis Centers. All these data are publicly available[2]. The following steps are performed to obtain the final product "UT1-UTC" of the INTENSIVES:

1) Coordination of the observing program by the IVS Coordinating Center

2) Preparation of the schedules by the IVS Operation Centers

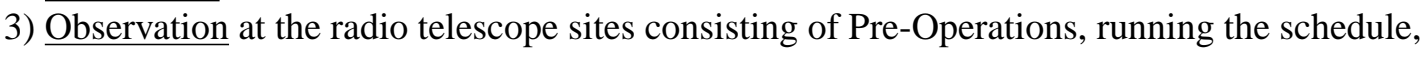
data acquisition and transport to the correlators

4) Correlation at one of the IVS Correlators

5) Data Analysis at the IVS Analysis Centers

6) Archiving the data at the Data Centers and providing products like Earth Orientation Parameter (EOP) time series, daily files in the Solution (software/technique) INdependent EXchange Format (SINEX) or specific analysis

7) Combination of the results by evaluating models, comparing solutions and calculating combinations of different VLBI solutions

By using the new e-transfer techniques, the time delay from the scheduled observation to the UT1-UTC time series is reduced to a few days now. E-transfer is used to transfer the observed raw data from the IVS Network Stations to the correlators after the observation is finished. For the INTENSIVES, standard internet connections offer transfer rates of up to $600 \mathrm{Mbit} / \mathrm{sec}$. Also the IVS Analysis Centers receive and send their data via electronic transfer from and to the data centers. This means that for the INTENSIVES physical shipment of disks is not needed anymore during the 
whole data production. It is a regular and proven usage of e-VLBI techniques for a daily production of the product UT1-UTC. It is a pre-stage to meet the goals for a new generation of VLBI systems. These goals are defined by IVS Working Group 3, which proposes continuous measurements of EOP within a rapid generation and distribution of this IVS product [3]. Already with the current technology it should be possible to transfer data in near real-time during the experiment. Emerging internet capabilities will further help to reach this goal.

\section{Geometry of the INTENSIVES network and its coordination}

The INT1 sessions last one hour to allow quick data transport with "standard" internet equipment. They are observed from Monday to Friday together with Kokee Park Geophysical Observatory (Hawaii, USA) and every other Thursday also with the radio telescope Svetloe (Russia). The data are correlated at Washington Correlator. On Saturday and Sunday Wettzell (Germany) and Tsukuba (Japan) are engaged in the INT2 sessions which are correlated in Japan. On Monday morning INT3 completes the INTENSIVE observations in the network of Wettzell, Tsukuba and Ny Ålesund (Spitsbergen, Norway). The INT3 sessions are correlated in Bonn. Fig. 1 shows the geometry of the INTENSVIVES network.

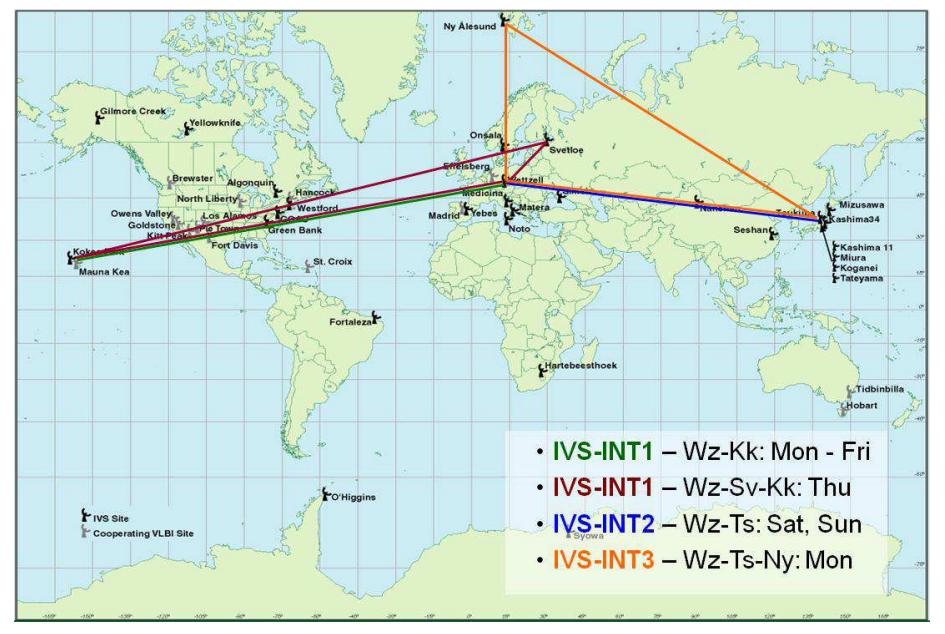

Figure 1: Geometry of the INTENSIVES network [5]

The INTENSIVES' schedules are optimized for the boundary conditions of a one-hour experiment to determine dUT1=UT1-UTC and offer one-way observations with a homogeneous sky coverage. The accuracy of the final dUT1 estimation depends on the east-west-distance of the baseline while low elevations allow it to estimate the troposphere parameters. In this case the sky coverage is limited because only a few sources are simultaneously visible at participating sites. All these conditions must be optimized with respect to the possible observing time per source. They determine the schedule offered by the IVS Operation Centers.

\section{Observation and data transfer at Wettzell}

At Wettzell the standard equipment for S- and X-band is used for INTENSIVES. The intermediate frequency offered by the cryogenic low noise receiver is split into 14 small bands by video 
converters via an intermediate frequency distributor. A 1-bit formatter digitizes the signals for data acquisition with a Mark 5a (Mk5a) system to record at up to $1 \mathrm{Gbit} / \mathrm{sec}$ on disk packs (hard-disk arrays consisting of eight commodity hard-disk drives). After the recording with Mk5 the data scans must be manually copied onto a local harddrive of a specific Mk5a system for e-VLBI which is located outside the firewall. In addition to the Mk5 recording, the bit stream can also be recorded in parallel with EVN-PCs on a local harddrive with an Unix file system at $512 \mathrm{Mbit} / \mathrm{sec}$ or via a mounted Network File System directory to a RAID-system at $400 \mathrm{Mbit} / \mathrm{sec}$. The RAID system offers effective capacities of up to 60 TByte. From these EVN-PCs, the correlators can transfer data without additional copying.

The Wettzell antenna can be operated in three observational modes. The standard mode is that a local operator starts and controls the observation. To reduce the burden of weekend shifts the antenna can also be run in an unattended mode without a local operator. In case of errors, responsible contact persons are called by the local security guards. This mode works quite reliably as long as the equipment is properly maintained and a local safety concept is implemented. The third mode has been made possible by software for remote control and attendance. With this, an operator gets almost complete remote access via internet to the RTW's NASA Field System which controls the observation. The option of remotely controling the antenna is currently in a test phase [4].

Wettzell is connected to the internet via fiber and has a $622 \mathrm{Mbit} / \mathrm{sec}$ connection at the moment. Behind the gateway router the local area network is $1 \mathrm{Gbit} / \mathrm{sec}$. The locally saved observation data are fetched by the correlators directly after the observations. Washington uses "bbFTP"[7] to electronically transfer data from the dedicated e-VLBI Mk5a at data rates of up to $70 \mathrm{Mbit} / \mathrm{sec}$. The data used to be sent to a node close to the Washington Correlator over the network. Then the data disk packages had to be carried over to the correlator ("sneaker net"). Now, however, data can be transfered to the correlator directly. The correlator at the Geographical Survey Institute (GSI) in Tsukuba uses the "Tsunami" protocol[8] for downloading at a rate of 300 to $400 \mathrm{Mbit} / \mathrm{sec}$ between Germany and Japan. In a subsequent step the data must be converted from Mk5 to the Japanese K5 system, which can be used by the Tsukuba software correlator. Further the correlator of the MaxPlanck-Institute für Radioastronomie Bonn also uses Tsunami for the connection within Germany at rates of $500 \mathrm{Mbit} / \mathrm{sec}$. Bonn currently has a $1 \mathrm{Gbit} / \mathrm{sec}$ connection. In summary, it means that the data of the one-hour session can be sent to the correlators in much less than one hour.

\section{Correlation and data analysis at BKG}

The correlation in Washington and Bonn is done on hardware correlators. Tsukuba uses a software correlator for the INTENSIVES. But the conversion to K5-system is still necessary [6]. After the correlation the resulting data are sent to the IVS Data centers from where the IVS Analysis Centers download them for processing the analysis. They produce quarterly solutions for TRF and CRF and continuously provide troposphere parameters, e.g. wet zenith delay. In combination with the INTENSIVES they offer continuous EOP time series using 24 hours and 1 hours observations (Bulletin B) and supply daily SINEX files for it. BKG also writes the schedules for the INT2 sessions using the baseline Tsukuba-Wettzell [5]. To improve the results of the analysis, the software is being continuously enhanced. 
At BKG, a mixture of semi-automated and interactive processing is used in the analysis. The data arrive on a publicly accessible data server outside the firewall. Semi-automated procedures take care of mirroring, filtering and doing the pre-analysis to prepare the data for the actual processing. After that the data are copied into the internal database system where an interactive processing with Calc/Solve is used for outlier reduction, to treat ambiguities, to do the weighting and parametrization and to detect clock jumps. As this process can hardly be automated, an operator is needed to make the final decisions. A following semi-automated post-procedure creates the IVS products and posts the final results to the IVS Data Centers. The whole process is monitored and can be controlled via a connected information database. The resulting time series can be used to combine different VLBI solutions which improves the results.

\section{Summary and outlook}

The offered IVS product UT1-UTC closes the cycle from scheduling the INTENSIVES, to observation, to analysis where the UT1-UTC time series are produced. It defines a routinely offered product where e-VLBI techniques are regularly used in a daily process. These new techniques reduce the production time to a few days so that highly accurate products can be offered in a timely fashion. These data again form an essential basis for other geo-oriented, scientific observations or practical applications such as high-precision navigation and positioning. To improve these production times further e-VLBI real-time communication tests are planed whereby the transfer is done during the observation so that local recording is no longer needed. A first test with Washington is planned before the end of the year as a feasibility study.

\section{References}

[1] IVS, About IVS. IVS Terms of Reference, http://ivscc.gsfc.nasa.gov/about/org/ documents/ivstoR.html\#dc (2009).

[2] IVS, Components. Data Centers, http://ivscc.gsfc.nasa.gov/about/org/ components/dc-list.html (2009).

[3] A. Niell, et. al., VLBI2010: Current and Future Requirements for Geodetic VLBI Systems, ftp://ivscc.gsfc.nasa.gov/pub/annual-report/2005/pdf/spcl-vlbi2010.pdf (2009).

[4] A. Neidhardt et. al., e-control: new concepts for remote control of VLBI-telescopes and first experiences at Wettzell, in proceedings of The 8th International e-VLBI Workshop, EXPReS09, POS (EXPReS09) 038 (2009).

[5] W. Schwegmann, A. Neidhardt, et. al., VLBI in near real-time: Von der Beobachtung zu kombinierten Produkten am Beispiel der INTENSIVES, Geodätisches Kolloquium BKG Frankfurt a. Main (2009).

[6] K. Takashima, K. Nozawa, Japanese Software Correlator for UT1 Intensive, http://www.fs.wettzell.de/veranstaltungen/vlbi/frff2009/SW_CORRELATOR/ Japanese_correlator_V2C.pdf (2009).

[7] bbFTP, http://doc.in2p3.fr/bbftp/ (2009).

[8] sourceforge.net, Tsunami UDP Protocol, http://t sunami-udp. sourceforge. net/ (2009). 